Cahiers de recherches médiévales

\title{
La Vita beati Hysidori de Juan Gil de Zamora
}

Editio princeps

Jose Carlos Martín

\section{CpenEdition}

\section{Journals}

Édition électronique

URL : https://journals.openedition.org/crm/10742

DOI : $10.4000 / \mathrm{crm} .10742$

ISSN : $1955-2424$

Éditeur

Honoré Champion

Édition imprimée

Date de publication : 10 décembre 2008

Pagination : 119-133

ISSN : 1272-9752

Référence électronique

Jose Carlos Martín, "La Vita beati Hysidori de Juan Gil de Zamora », Cahiers de recherches médiévales [En ligne], 16 | 2008, mis en ligne le 15 décembre 2011, consulté le 15 décembre 2022. URL : http:// journals.openedition.org/crm/10742; DOI : https://doi.org/10.4000/crm.10742 


\title{
酷M
}

\section{La Vita beati Hysidori de Juan Gil de Zamora Editio princeps*}

\begin{abstract}
This paper offers the editio princeps of the Vita beati Hysidori from the Legendae sanctorum et festiuitatum aliarum by the Franciscan monk Juan Gil de Zamora ( $+c a$. 1318). We know only one manuscript of this hagiographical collection: London, British Library, Add. 41070 (XIVth Century). The main sources of the Vita b. Hysidori are the Chronicon Mundi of Lucas de Tuy, the Vita s. Isidori (BHL 4486), the Translatio s. Isidori Legionem anno 1063 (BHL 4488), the Historia translationis s. Isidori (BHL 4491) and the Historia de rebus Hispanie of R. Jiménez de Rada.
\end{abstract}

Résumé: Cet article propose l'édition princeps de la Vita beati Hysidori extraite des Legendae sanctorum et festiuitatum aliarum du franciscain Juan Gil de Zamora ( $+c a$. 1318). On connaît un seul manuscrit de cette compilation hagiographique: London, British Library, Add. 41070 (XIV siècle). Les principales sources de la Vita b. Hysidori sont le Chronicon Mundi de Lucas de Tuy, la Vita s. Isidori (BHL 4486), la Translatio s. Isidori Legionem anno 1063 (BHL 4488), l'Historia translationis s. Isidori (BHL 4491) et l'Historia de rebus Hispanie de R. Jiménez de Rada.

Élaborer une editio princeps est une tâche souvent ardue mais qui rend service à la communauté scientifique. Tel est le but du présent travail dans le domaine précis de la réception d'Isidore de Séville $(† 636)$ durant le Moyen Âge tardif'.

Le franciscain Juan Gil de Zamora († ca. 1318) fut un auteur très prolifique 2 . Ses Legendae sanctorum et festiuitatum aliarum (BHL-, DIAZ-) sont une compilation de vies de saints classées par ordre alphabétique, composée vers 1282 , et dont seul le prologue a été édité

\footnotetext{
* Travail assigné aux Projets HUM2006-05744/FILO (DGICYT) et SA103A05 (JCYL).

${ }^{1}$ Sur les compositions hagiographiques hispano-latines du Moyen Âge en l'honneur d'Isidore de Séville, voir J. C. Martín, «El corpus hagiográfico latino en torno a la figura de Isidoro de Sevilla en la Hispania tardoantigua y medieval (ss. VII-XIII)», Veleia, 22, 2005, p. 187-228.

${ }^{2}$ Sur sa vie, voir M. de Castro y Castro, Fray Juan Gil de Zamora, O. F. M., De preconiis Hispanie, Madrid, Universidad de Madrid, 1955, p. XXXV-CXXVI. Sur sa production littéraire, voir surtout M. de Castro y Castro, «Juan Gil de Zamora. Ciencia y sabiduría », Verdad y Vida, 57, 1999, p. 371-376; voir aussi : K. Reinhardt et H. Santiago-Otero, Biblioteca Bíblica Ibérica Medieval, Madrid, Consejo Superior de Investigaciones Científicas, 1986, p. 194-200 ( $\left.{ }^{\circ} 84\right)$; et H. Santiago-Otero, «Juan Gil de Zamora : Comentarios a la Biblia », Amar, sentir e viver a História - Estudos de Homenagem a Joaquim Veríssimo Serrão, Lisboa, Edições Colibri, 1995, t. 1, p. 435-443.

${ }^{3}$ F. Dolbeau, "Les prologues de légendiers latins", Les prologues médiévaux. Actes du Colloque international organisé par l'Academia Belgica et l'École française de Rome avec le
}

Cahiers de Recherches Médiévales, 16, 2008 
La tradition manuscrite de cette œuvre se réduit à un manuscrit : London, British Library, Add. 41070, du XIV ${ }^{\mathrm{e}}$ siècle et d'origine hispanique ${ }^{4}$, qui contient uniquement l'ouvrage cité de J. Gil. À la lettre H, aux fol. $199^{\mathrm{v}}-210^{\mathrm{r}}$, il comporte une Vita b. Hysidori, dont les sources sont le Chronicon Mundi de Lucas de Tuy Vita s. Isidori (BHL 4486) ${ }^{6}$, la Translatio s. Isidori Legionem anno 1063 (BHL 4488), l'Historia translationis s. Isidori (BHL 4491) ${ }^{8}$, et l'Historia de rebus Hispanie de R. Jiménez de Rada'. Contrairement à ce que j'ai écrit dans un travail antérieur ${ }^{10}$, l'utilisation de l'Adbreniatio Braulii Caesaraugustani ep. de uita s. Isidori (CPL $1215)^{11}$ n'est pas certaine, car tous les passages qui sont communs avec cette œuvre proviennent sûrement, à l'exception d'un seul, du Chronicon mundi de Lucas de Tuy.

Critères de cette édition : étant donné que le texte de J. Gil est une sorte de centon, là où il suit de près sa source, je corrige à partir de celle-ci les constructions qui me paraissent de pures erreurs de copie, tandis que je maintiens les variantes qui offrent un texte intelligible; au contraire, quand c'est J. Gil lui-même qui écrit, résumant ses sources ou ajoutant des expressions qui lui sont propres, je laisse le texte tel quel, même s'il s'éloigne des usages attendus en latin.

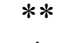 \\ Sigles : \\ $L=$ London, British Library, Add. 41070, fol. $199^{\mathrm{v}}-210^{\mathrm{r}}$.
}

\footnotetext{
concours de la FIDEM (Rome, 26-28 mars 1998), éd. J. Hamesse, Turnhout, Brepols, 2000, p. $345-393$ : p. 373.

${ }^{4}$ Consulté sur microfilm. La meilleure étude sur ce manuscrit est due à J. Pérez-Embid Wamba, Hagiología y sociedad en la España medieval. Castilla y León (siglos XI-XIII), Huelva, Universidad de Huelva, 2002, p. 307-322. Autres références dans J. C. Martín, «El corpus hagiográfico latino... », art. cit., p. 193 n. 23.

${ }^{5}$ Édition : E. Falque, Lucae Tudensis Chronicon mundi, Turnhout, Brepols, 2003 (СCCM 74).

${ }^{6}$ Édition partielle dans les Acta Sanctorum Aprilis collecta... Tomus I. quo priores $X$ dies continentur, Antverpiae, apud Michaelem Cnobarum, 1675, p. 330-351. Les passages non édités par les Bollandistes ( $\$ 19 a-b)$ peuvent se lire dans J. C. Martín, «El corpus hagiográfico latino... », art. cit., p. 227-228.

${ }^{7}$ Édition: F. Arévalo, S. Isidori Hispalensis episcopi Hispaniarum doctoris opera omnia, 7 vol., Romae, Typis Antonii Fulgonii, 1797-1803 : vol. 1, p. 40-44 (= PL 81,39D-43C). Autre édition : F. Santos Coco, Historia Silense, Madrid, Centro de estudios históricos, 1921, p. 93 99.

${ }^{8}$ Édition: J. A. Estévez Sola, dans L. Charlo Brea, J.A. Estévez Sola et R. Carande Herrero, Chronica Hispana saeculi XIII, Turnhout, Brepols, 1997 (CCCM 73), p. 143-179.

${ }^{9}$ Édition: J. Fernández Valverde, Roderici Ximenii de Rada Historia de rebus Hispanie siue Historia Gothica, Turnhout, Brepols, 1987 (СССм 72).

${ }^{10}$ J. C. Martín, «El corpus hagiográfico latino... », art. cit., p. 193 et 219-222.

${ }^{11}$ Édition: A. E. Anspach, Taionis et Isidori nova fragmenta et opera, Madrid, Centro de estudios históricos, 1930, p. 57-64.
} 
adbr. : Adbreuiatio Braulii Caesaraugustani ep. de uita s. Isidori.

chron. : Lucas de Tuy, Chronicon mundi.

hist. reb. : R. Jiménez de Rada, Historia de rebus Hispanie.

hist. trans. : Historia translationis s. Isidori.

Leg. : Translatio s. Isidori Legionem anno 1063.

uit. Isid. : Vita s. Isidori (BHL 4486).

\section{Vita beati Hysidori}

Incipit uita beati Hysidori.

Hysidori doctoris uenerabilis sacram ystoriam describentes, primo agemus de ipsius genere ac conuersacione in statu seculari ; secundo, in statu pontificali ; tercio, de ipsius consumacione finali; quarto, de translacione; quinto de miraculorum operacione $^{12}$.

1. (1) ${ }^{13}$ Hysidorus archiepiscopus Yspalensis et doctor egregius Carthaginensis extitit nacione ac filius Seueriani ducis eiusdem prouincie, qui extitit filius Theodorici regis Gothorum. Genuit siquidem Seuerianus, filius regis Theodorici, ex Theodora uxore sua, filia Sisebuti regis, illos tres inclitos Leandrum, scilicet archiepiscopum Yspalensem, et Hysidorum archipresulem, ambos primates Yspanie, atque Fulgencium, episcopum Astigitanum. Omnes igitur conffessores filios meruit optinere. Florentina, quoque religiosarum abbatissa, et Theodosia, gloriosa regina, sanctorum horum doctorum ex utroque parente sorores fuerunt.

$(2)^{14}$ Quales hii doctores fuerint, eorum florigeris libris et beneficiis orbis experitur cotidie uniuersus. Laudabilis est inter sacras uirgines illa sanctissima Florentina, sed dignis preconiis offerenda ${ }^{15}$ est gloriosissima Theodosia, soror Hysidori, Leandri et Fulgencii et etiam Florentine. Etenim ipsa in sanctissimo Hermegildo $^{16}$ rege et martire, filio suo, acerrimam mentis pertulit passionem. Fuit autem Theodosia uxor Leouigildi et ex eo suscepit Hermegildum et Recharedum, nepotes Hysidori prelibati.

(3) ${ }^{17}$ Prefatus uero Ysidorus, cum esset infantulus et a nutrice ad ortum inter brachia portaretur, ibidem obliuione ducta eundem infantem inter olera dereliquid. Post aliquos autem dies Seuerianus lugens filium suum solium ascendit et, aspiciens contra uiridarium, uidit innumeram apum multitudinem et, uocatis cunctis

\footnotetext{
${ }^{12}$ J'ai divisé le texte de mon édition en cinq chapitres conformément au plan de J. Gil en cinq parties.

${ }^{13}$ Source : chron. praef. 2 1. 37-43.

${ }^{14}$ Sources : quales - passionem chron. praef. 2 1. 43-48; Theodosia-Recharedem chron. 2,68 1. $2-4$.

${ }^{15}$ offerenda : efferenda chron. praef. 2 l. 46 (sed lectio offerenda legitur in apparatu).

${ }^{16}$ Hermegildo scripsi cum fonte, Hermogillo $L$.

${ }^{17}$ Source : uit. Isid. 3.
} 
seruentibus, festinauit ad ortum ut rei geste uideret miraculum. Accedentes autem uiderunt alias apum in os pueri intrantes et exeuntes alias, faciem ${ }^{18}$ uero et totum corpus melli et faui operimenta texentes. Cum uero pater ${ }^{19}$ filium cum clammore et lacrimis amplectetur, apes in tantam se altitudinem extulerunt ut oculis corporeis non possint uideri.

$(4)^{20}$ Cum autem fuisset literis traditus et minus capacis ingenii floreret, ut sibi uidebatur, uerbera magistri metuens, fugiit ab Yspalense urbe. Cumque fatigatus iuxta marginem cuiusdam putei siciens resedisset, aspexit quoddam saxum tortuosis foraminibus perforatum. Similiter in ore putei erat quoddam lignum continuis cordarum seccionibus in aquis hauriendis canalatum. Quibus uisis, cogitare cepit quis illorum foraminum in lapide et illarum concauitatum in ligno fuerit operator.

$(5)^{21}$ Dum hec secum tacitus cogitaret, uenit mulier ad hauriendam aquam, que, pulcritudinem pueri admirata, interrogauit quid illic ageret aut ${ }^{22}$ cur ita solus huc aduenisset. Ad quam puer: O domina, rogo ut mihi dicas quis uel ad quot lapidis huius foramina uel istius ligni canales facti fuerint. Cui mulier: Lapis iste, ait, frequenti stillacione gutarum fuit perforatus, et lignum hoc frequenti cordarum ductu in hauriendis aquis extitit canalatum. Puer, hoc audito, ait: Et si lapis durissimus mollis aque frequenti stillacione cauatur et lignum cordarum seccionibus cedit, quanto magis ego homo, Dei gracia preueniente, discendo cotidie paruissimis incrementis, ualeam ad augmentum sciencie peruenire.

(6) ${ }^{23}$ Posthec puer concito $^{24}$ reuersus Hyspalim se magistrorum humili deuocione subdidit disciplinis. Cui Deus contulit tantam graciam ut Platonem ${ }^{25}$ ingenio, Aristotilem studio, eloquencia ${ }^{26}$ Tullium, erudicione Origenem, grauitate Augustinum, doctrina Gregorium prosequeretur exemplis.

$(7)^{27}$ Processu uero temporis, cum beatus Gregorius haberet multum familiarem beatum Leandrum, fratrem Ysidori, episcopum Yspalensem - ob amore eius etiam librum Moralium composuit-, desiderabat multum uidere Hysidorum, fratrem Leandri. Cum Ysidorus eius desiderium apperisset, quod mirabile est auditu, nocte natalis Domini in ecclesia Yspalensis, prima leccione perlecta, extra ecclesiam egressus, nescio a quo ductus in paruissimo temporis interuallo ad Romanam deuenit urbem atque beatum Gregorium in Natiuitatis laudibus Deo psallentem inuenit. Viso eo, beatus Gregorius nimis letus protinus agnouit atque amicissimum sibi Leandrum in Ysidoro amplexatus est. Post euangelicam uero explecionem ualefaciens sancto, ab ecclesia egressus, Hyspalim in ipsa nocte rediit et clericos quos dimiserat $\mathrm{ea}<\mathrm{S}>\mathrm{dem}^{28}$ matutinas laudes reperit celebrantes. Quod uero hoc ${ }^{29}$ sanctissimi

\footnotetext{
${ }^{18}$ ante faciem legitur super in fonte.

${ }^{19}$ pater scripsi cum fonte, propter $L$.

${ }^{20}$ Source : uit. Isid. 4.

${ }^{21}$ Source : uit. Isid. 4.

${ }^{22}$ aut scripsi cum fonte, ait $L$.

${ }^{23}$ Sources : posthec - disciplinis uit. Isid. 4 ; cui - exemplis uit. Isid. 5

${ }^{24}$ post concito legitur gressu in fonte.

${ }^{25}$ Platonem scripsi cum fonte, plantacionem $L$.

${ }^{26}$ eloquencia scripsi cum fonte, oloquencia $L$.

${ }^{27}$ Source : uit. Isid. 6.

${ }^{28}$ easdem scripsi cum fonte, eadem $L$.
} 
Gregorii obtentu uel Hysidori meritis aut aliquo alio modo factum fuerit, fateor me ambigere.

$(8)^{30}$ Era uero D[III]C $C^{\mathrm{a}}{ }^{31}$ XXVIII ${ }^{\mathrm{a}}$. anno quarto imperii Marciani ${ }^{32}$ Leouigildo $^{33}$ deffuncto, filius eius Recharedus, quem Leander, frater Ysidori, conuerterat ad fidem, in regno est coronatus. Qui de diuersis Yspanie ac Gallie prouinciis sinodum episcoporum congregans, condempnauit heresim arrianam, quam deffensauerat pater suus. Interfuit tunc dignitate primas ortodoxus Leander, archiepiscopus Yspalensis et ecclesie Romane legatus.

$(9)^{34}$ Qui cum heresis arriane feruorem [et] $]^{35}$ tempore Leouigildi ab Yspanis quondam confutare radicitus nequisset, pro confirmandis sancte et inmaculate Trinitatis capitulis ad Constantinopoli $<\operatorname{tanu}>$ m presulum $<$ cetum $>^{36}$ transmeauit. Ibi adherat beatus Gregorius doctrina et sanctitate clarissimus, tunc cardinalis, uices Romani antistitis agens. Cum quo ipse Leander amicicie fedus pepigit et ab eo postulauit ut librum beati Iob sibi exponeret, quod postea ille deuota mente compleuit.

$(10)^{37}$ Leander uero postquam ad Yspanias rediit, Gotis predicando suasit ut Patrem et Filium et Spiritum sanctum trinum secundum personarum distinccionem et unum secundum nature ${ }^{38}$ diuinitatem Deum esse crederent. Illis credentibus prior dedit precepta et ecclesiastica officia quibus fruerentur. Ipso namque tempore per eum $^{39}$ unda baptismatis loti Galleciam optinent ${ }^{40}$.

$(11)^{41}$ Leander uero post multos labores quos pro fide Christi pertulit, ecclesia pace adepta, spiritum celo reddidit. Doctor etiam eximius Gregorius Papa migrauit ad Dominum.

2. (1 $)^{42}$ Hysidorus uero, beati germanus Leandri, eidem in archiepiscopatu successit. Qui cum de Romana urbe, ad quam uocatus fuerat, redisset, ad Galliarum fines apropinquans mirabiliter a Domino magnificatus est. Cum enim Galliarum et Yspaniarum prouincie siccitate atrocissima laborarent, ita ut fruges et herbe et arbores arescerent et homines ex ipsa aeris intemperie laborarent, audientes sancti antistitis aduentum, ei cum processionibus ocurrerunt rogantes ut pro eis Dominum precare[n]tur.

\footnotetext{
${ }^{29}$ hoc scripsi cum fonte, hic $L$.

${ }^{30}$ Sources : era - coronatus chron. 2,71 1. 1-4; qui-legatus chron. 2,72 1. 1-11.

${ }^{31} \mathrm{DC}^{\mathrm{a}}$ scripsi cum fonte, DIIICa $L$.

${ }^{32}$ Marciani : Mauricii chron. 2,71 l. 1.

${ }^{33}$ Leouigildo scripsi, Leouigillo $L$.

${ }^{34}$ Source : chron. 2,72 1. 12-19.

${ }^{35}$ et seclusi.

${ }^{36}$ constantinopolitanum - cetum scripsi cum fonte.

${ }^{37}$ Source : chron. 2,72 1. 19-25.

${ }^{38}$ nature scripsi cum fonte, abbreuiaturam pro nunc uel nec habet $L$.

${ }^{39}$ post eum legitur sueui in uit. Isid. 12.

${ }^{40}$ optinent scripsi cum fonte, optineret $L$.

${ }^{41}$ Source : chron. 2,72 1. 25-28.

42 Sources: hysidorus - successit uit. Isid. 15-16; qui- fuerat: uit. Isid. 18; redissetprecare[n]tur uit. Isid. 19.
} 
$(2)^{43}$ Ad cuius preces statim Dominus [ad cuius preces Dominus statim] $<$ post $\rangle^{44}$ repentinos ac fulgureos ${ }^{45}$ continuos ictus tantam dedit aquarum inundanciam ut quis non uideret temporibus retroactis huius pluuie comparacionem. Et omnes timore mortis exterriti ad ecclesiam confugerunt. Egroti uero, qui humeris ${ }^{46}$ baiulancium beati patris subsidia expectantes aduenerant, sue inmemores imbecillitatis etiam ceteros fugiendo precurrebant, nec inmerito, omnes enim beati patris optentu redditi erant pristine sanitati.

$(3)^{47}$ Congregatis uero ad eum populis, uisis tantis miraculis, ait sanctus : Ecce fratres quantum obtinet fides sanctissime Trinitatis. Tria enim uobis a Domino per peticionem uestram beneficia sunt collata, scilicet, sanitas cordis, temperies aeris et exuberacio frugum ac fructuum habundancia.

(4) ${ }^{48}$ Veniente autem eo procul ab Hyspalense ciuitate, Machometus qui tunc erat in Yspania, cognito eius aduentu, accelerauit fugam atque in Affricam ueniens maximam Ysmaelitarum multitudinem suis predicacionibus seduxit. Nam diabolus transfigurans se in angelum lucis apparuit Machometo dicens: O dilecte mi, aduersarius noster adest Hysidorus, nobis et omnibus nostris per omnia inimicus. Velociter ergo ab Hyspanie finibus abscede, quod eius presenciam nec tu nec angeli poterunt sustinere.

$(5)^{49}$ Apropinquante ergo beato Hysidoro Hyspalim et accedente ad locum qui Sancta Eulalia uocatur, apparuit horride uisionis inmanissima ${ }^{50}$ belua, ex cuius ore quasi inundantis torrentis rapidissimi cum flammarum crepitu sonus audiebatur. Ortabatur uero suos sanctus ut, depulso timore, muniti redempcionis signo audacter accederent. Apropinquantibus illis, draco qui seuissimus ocurrebat, demisso capite, stetit, quasi preceptum sustinens imperantis. Cui sanctus: In nomine, ait, Ihesu Christi, fili Dei, precipio tibi ut eas in locum ubi nulli noceas creature. Ad hoc serpens cum sibilis et strepitu recedens numquam comparuit.

$(6)^{51}$ Cum uero totus populus Yspalensis exiret obuia ei, ex oppressione turbarum quedam mulier $<$ filium $>^{52}$ habens in utero prefocata spiritum exalauit. Quod sanctus cernens et flens ubertim, uerba orationis ad Deum tacitus fudit. Post aliquantum uero temporis spacium mulier surrexit clamans uoce magna, dicens : Beatus tu, pater sancte Ysidore, et beatus sermo oris tui.

$(7)^{53}$ Populus autem conuersus ad mulierem scicitabatur ${ }^{54}$ qualiter accidisset ei. Ad quos illa: Ingrediente, inquid, anima fili mei in utero de corpore, caterua demonum affuit, qui nexibus igneis nos ad penarum loca rapere parabant. Sed

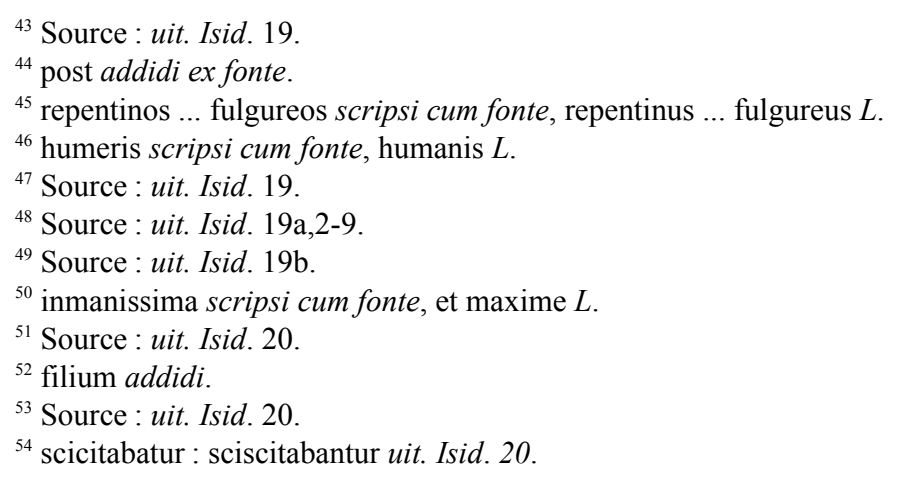


gloriosissimo patre nostro pro nobis orante, audita est uox dicens: Reuertantur anime eorum in corpora sua, quoniam amicus Dei Hysidorus orat pro eis. Quo audito, unus angelorum qui semper fuit cum beatissimo doctore Hysidoro nos ad corpus reduxit. Eleuans itaque omnis populus uocem in celum, laudes clarius Altissimo personando, eum in urbem duxerunt.

$(8)^{55}$ Processu uero temporis, cum disputasset cum quodam heretico, nomine Gregorio, et eum uicisset, ita quod ipse hereticus uictum se coram omnibus testaretur, cecus quidam astitit importunis uocibus conquerendo: Heu, inquid, magne doctor Ysidore, diu est quod de tua sanctitate percipi famam, sed ecce tibi assistens, bonitate tua priuatus, te uidere non mereor. Intuitus autem [unum] eum predictus Gregorius, beatum dissimulantem Hysidorum precabatur ut pontificalem cirotecam, quam manu tenebat, porrigeret ${ }^{56}$. Qua impetrata, in conspectu omnium fidus surrexit et, [de] ceci occulos de ea tangens, clara uoce dixit: Dominus Ihesus Christus, qui per beatum Hysidorum cordis mei illuminauit occulos, corpus tuum ad confirmacionem fidei dignetur illuminare. Quo dicto, de palpebris ceci sanguine erumpente, uisum recepit.

3. (1 $)^{57}$ Hysidorus ergo doctor Hyspaniarum anno VI. regni Cintilliani regis, dum esset in ecclesia, peracto sermone ad populum et expandens manus ad celum ac benedicens omnibus, Deo sibi actenus traditum gregem comendans, felicissimo sompno obdormiuit in Domino senio sanctissimo decoratus.

$(2)^{58}$ Extitit quippe spiritu prophecie clarus, in elemosinis largus, in hospitalitate precipuus, corde serenus, in sentencia uerax, in iudicio iustus, in predicacione strenuus, in exortacione letus, in lustrandis Deo animabus studiosus, in exposicione Scripturarum cautus, in consilio prouidus, in habitu humilis, in mensa sobrius, in oracione deuotus, honestate preclarus, semper pro ecclesia ac ueritatis deffensione ${ }^{59}$ pronus et omni bonitate conspicuus. Preterea pater extitit clericorum, doctor et sustentator monacorum ac monialium, consolator merencium, tutamen pupillorum ac uiduarum, leuamen oppressorum, deffenssor ciuium, atritor superborum, persecutor et malleus hereticorum.

$(3)^{60}$ Rexit archipresulatum Yspalensem X. ${ }^{61}$ annis, diuersis fulgens miraculorum signis, primacie dignitate florens et Romani Pape in Hyspaniis uices gerens. Sacerdotibus, regibus et populis diuinas et humanas leges tradidit, et Romano antistiti humiliter obedire precepit. Nolentibus obedire maledictionem intulit et eos a fidelium consorcio sepparauit. Multis librorum uoluminibus Christi ecclesiam informauit et in pace quieuit. Era $\mathrm{DC}^{\mathrm{a}}$. LXX ${ }^{\mathrm{a}}$. Toledo ad sinodum episcopos conuocauit et ore prophetico eis sui obitus diem et Yspanis uentura predixit.

\footnotetext{
${ }^{55}$ Source : uit. Isid. 27-28.

${ }_{56}^{56}$ ante porrigeret legitur sibi in uit. Isid. 28.

${ }^{57}$ Source : chron. 3,3 1. 5-9.

${ }^{58}$ Source : chron. 3,3 1. 9-20.

${ }^{59}$ post deffensione legitur mori in chron. 3,3 l. 15.

${ }^{60}$ Source : chron. 3,3 1. 20-28.

${ }^{61}$ X. : XL. chron. 3,3 l. 20.
} 
$(4)^{62}$ Dormiuit igitur beatus Ysidorus cum patribus suis era DC $C^{\mathrm{a}}$. LXX ${ }^{\mathrm{a}}$, , anno ab incarnacione Domini DC. XXII. Pro cuius obitu tam clerici quam laici non modicum sunt tristati, sed non multo post luctus in gaudium est conuersus. Nam de corpore eius tanta erupit fragrancia, omnium aromatum uincens odorem, ut omnes qui adhera $<\mathrm{n}>\mathrm{t}^{63}$ celesti uiderentur perfrui beatitudine. Diuersarum egritudinum ocurrunt infirmi, qui non solum san $<\mathrm{ct}>\mathrm{i}^{64}$ corporis tactu, uerum etiam solo odore redduntur pristine sanitati.

$(5)^{65}$ Interea plures fuere diuino illuminati dono, qui uiderunt sancti animam in sublime $\mathrm{ab}$ angelorum multitudine in uoce laudis et iubilacionis defferri, et Christum, filium Dei, cum innumerabili caterua sanctorum ei obuiam procedentem.

(6) ${ }^{66}$ Obiit autem beatus conffessor Ysidorus sub die nonarum aprilis, temporibus cristianissimi Cintilliani regis Yspanie, et Eraclei imperatoris Romani, qui crucem dominicam, quam impius rex Cosdroe asportauerat, loco suo Iherosolimis constituit ad laudem almiflui Ihesu Christi, cui est honor et gloria per secula infinita. Amen.

4. $(1)^{67}$ Ysidori beatissimi corpus gloriosum postquam fuit traditum sepulture, gentes Gothorum occulto Dei iudicio gentili gladio traditur ferienda ${ }^{68}$. Anno ab incarnacione Domini $<\ldots .>>^{69}$, transmarini namque Arabes Yspalim uenientes, eadem urbem miserabiliter eruperunt, deinde Beticam et Lusitaniam prouinciam ocupantes, exceptis montibus Asturiarum, Alaue, Bizcaye et Ruconie, loca sancta et res sacras profanauerunt, quousque paulatim et post longum tempus eos rex Pelagius et alii uiri catolici ab ocupatis sedibus abegerunt.

(2) Surrexit uero in posterum rex Fernandus, filius regis Sancii, monarcha Hyspanie interioris, scilicet, Yberi, Asturiarum et Galacie, quia terre Nauarre inter Yberum et Pireneum remansera $<\mathrm{n}>\mathrm{t}^{70}$ Sancio, filio regis Garsie, qui fuit occisus in Penaleny.

$(3)^{71}$ Hic preffatus gloriosissimus rex Ffernandus ut atigit ceptra patris, sicut in nostris ystoriis est digestum, inter reliqua opera pietatis que religiose gessit, petiit ab Abenabeth, Yspalensis urbis rege, quatenus corpus beatissime uirginis Iuste, quod in eadem urbe quiescebat concederet ut illum ad urbem Legionensem transire ferret $^{72}$. Cuius peticionibus, ut uoluit, assensum prebuit et ei se daturum quod petiit repromisit.

\footnotetext{
${ }^{62}$ Sources : dormiuit - DC. XXII. uit. Isid. 37 (cf. tamen adbr. 1. 135-137); pro cuius - sanitati uit. Isid. 36.

${ }^{63}$ adherant scripsi cum fonte, adherat $L$.

${ }^{64}$ sancti scripsi cum fonte, sani $L$.

${ }^{65}$ Source : uit. Isid. 37.

${ }^{66}$ Source : uit. Isid. 40

${ }^{67}$ Source : Leg. 1.

${ }^{68}$ ferienda scripsi cum fonte, facienda $L$.

${ }^{69}$ annus ab incarnatione Domini deest in $L$.

${ }^{70}$ remanserant scripsi, remanserat $L$.

${ }^{71}$ Source : Leg. 2.

${ }^{72}$ transire ferret : transferret Leg. 2.
} 
$(4)^{73}$ Qua sponsione accepta, conuocauit rex Fernandus uenerabilem Aluitum, Legionis urbis episcopum, et Munionem comitem cum magno apparatu militum, et eos ad defferendum prefate uirginis corpus Yspalim misit. Qui Yspalim uenientes, causam sue legacionis regi Benebeth exposuerunt. Quibus ille ait: Scio plane me Domino uestro quod dicitis promisisse, sed neque ego neque aliquis ex meis uobis corpus quod queritis demonstrabit. Vos ipsi querite et inuentum tollite et abite.

$(5)^{74}$ Quo responso accepto, uenerabilis Aluitus episcopus secrete fuit socios allocutus : Vt cernitis, o socii, itineris nostri laborem nisi diuina miseracio dignatiua dispensacione reuelet, frustrati penitus recedemus. Necesse est, dilectissimi, ut ab altissimo opere credamus ${ }^{75}$ et ob triduo ieiuniis et oracionibus insistamus, quatinus diuina maiestas ocultum nobis sancti cordis thesaurum dignetur misericorditer reuelare.

$(6)^{76}$ Placuit cunctis assistentibus exortacio presulis et per triduum illud ieiuniis et oracionibus peregerunt ${ }^{77}$. Iam cum die tercia ocumbente sole uenerabilis presul Aluitus oracioni peruigil insistebat, cumque psalmos sequestratos in celula recitaret, sompno opprimitur. Apparuit ei quidam uir ueneranda canicie comptus, pontificali infula ${ }^{78}$ accinctus, talique eum uoce alloquitur: Noui ad hoc te cum sociis deuenisse $\operatorname{adhuc}^{79}$ : uobis cum corpus Iuste uirginis beatissime defferatis. Et licet non sit diuine uoluntatis ut hec ciuitas huius uirginis priuetur abscessu, tamen diuina bonitas uacuos nullatenus uos remitet. Corpus namque meum est donatum, quod quidem tollentes afferte et cum eo ad propria remeate. Quem cum uenerabilis uir Aluitus quis esset interrogaret, qui talia sibi monita profferebat ${ }^{80}$, ait: Ego sum Hyspaniarum doctor huiusque urbis antistes Hysidorus. Quo dicto ab oculis eius euanuit.

(7) ${ }^{81}$ Presul uero euigilans congratulans cepit Deum contencius exorare ut hec uisio, si ex Deo esset, sibi secundo et tercio monstraretur. Rursumque obdormiuit, $<\ldots\rangle^{82}$ rursumque disparuit. Expergefactus iterum pontifex alacrius trinam uisionis amonicionem a Domino implorabat. Qui dum obnixius Deum exoraret, tercio sompno arripitur, et uir supradictus ueluti antea apparuit, et que bis dixerat tercio replicauit, et uirga pastorali, quam manu tenebat, solum percussit locum in quo sanctum corpus quiescebat, dicens : Hic, hic, hic inuenies corpus meum. Et ne putes te fantastica uisione deludi, hoc tibi do signum : mox ut corpus meum super terram eduxeris, scito quod statim ad nos uenies exutus corpore hoc mortali. Quo dicto ab eis aspectibus est ablatus.

\footnotetext{
${ }^{73}$ Source : Leg. 2.

${ }^{74}$ Source : Leg. 3.

${ }^{75}$ opere credamus : opem quaeramus Leg. 3.

${ }^{76}$ Source : Leg. 3.

${ }_{77}^{77}$ peregerunt : exegerunt Leg. 3.

${ }^{78}$ infula scripsi cum fonte, insula $L$.

${ }^{79}$ adhuc : ut Leg. 3.

${ }^{80}$ profferebat scripsi, prefferebat $L$.

${ }^{81}$ Source: Leg. 4.

${ }^{82}$ post obdormiuit desunt nonnulla uerba in L (cf. Leg. 4).
} 
$(8)^{83}$ Euigilansque presul certus de tanta uisione et de sua letus uocacione, facto mane, dixit ad socios: Oportet nos, dilectissimi, diuinam omnipotenciam submissis uultibus adorare, qui consueta misericordia nos precessit et frustrari mercede nostri itineris non est passus. Diuino nutu prohibemus membra beate uirginis Iuste hinc ${ }^{84}$ abstrahere, sed non minora defferemus pignora, dum corpus beatissimi Ysidori, qui hac in urbe sacerdocio et presulis ministerio fungebatur totamque Yspaniam suo decorauit uerbo, opere et exemplo, fuimus delaturi. Hec ait, et eis seriatim uisionis ordinem patefecit.

$(9)^{85}$ Quod audientes Deo gratias retulerunt. Et ad regem Sarracenorum pariter euntes, cunca que gesta fuerant per ordinem intimarunt. Quibus auditis, licet infidelis, uirtute Dei perpendens, expauit eisque respondit: Et si Hysidorum uobis tribuo, cum quo hic remanebo? Sed quia fas non erat uiros tante auctoritatis spernere $^{86}$, dedit sentenciam ut membra conffessoris inquirerent.

$(10)^{87}$ Mira loquar, nam sepulcrum beati corporis dum quereretur, uestigium uirge, cum <qua $>^{88}$ beatus conffessor tercia percussione locum monumenti monstrauerat, in ipso solo repertum est. Quo detecto, tanta fragrancia emanauit ut capillos capitis et barbe omnium qui aderant ueluti nebule balsamique rore perffunderet.

$(11)^{89}$ Corpus uero beatum ligneo uasculo, quod ex iunipero erat factum, erat obtectum. Statim uero ut reseratum est, reuerendum uirum Aluitum egritudo corripuit et, $\mathrm{VII}^{\mathrm{a}}$. die accepta penitencia, iuxta condictum uisionis angelis sanctis, ut credimus, tradidit spiritum. Et omnis exercitus, accepta gleba beati Ysidori et corpore presulis Legionensis Aluiti ad regem Ferdinandum repedare festinabant ${ }^{90}$.

$(12)^{91}$ Dum uero corpus beati Ysidori in lignum gestatorium poneretur, rex Sarracenorum supradictus Benebeth cortinam olosericam miro opere contextam supra corpus eius iactauit et magna ex intimo corde trahens suspiria, dixit: Ecce recedis hinc, Ysidore uenerande, ipse tamen nosti qualiter tua et mea pariter res agatur, unde precor mei memor sis.

$(13)^{92}$ Quibus peractis, ad propria cum summa leticia sunt reuersi. In quorum aduentu gloriosissimus rex Fferdinandus exhibuit ${ }^{93}$ apparatum, et licet contristaretur de obitu Aluiti presulis, quem mire semper fuerat ueneratus dilectione, tamen occursu $^{94}$ Hysidori ambiciosam < prebuit $>95$ pompam corpusque beati in basillica Iohannis Babtiste, quam idem rex nouiter fabricauerat, deposuit. Congregatisque

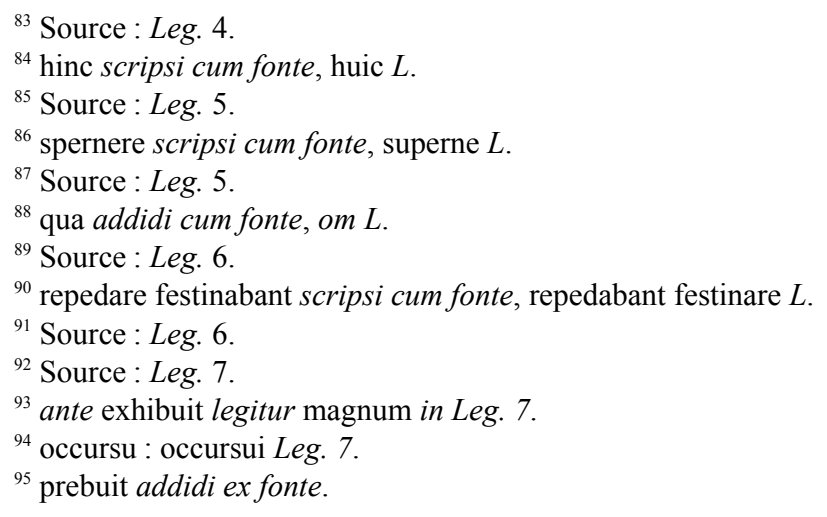


uniuersis regni sui nobilibus uiris atque episcopis, eam in honorem conffessoris consecrari fecit. Et ex tunc decimo kalendarum ianuarii dies dedicacionis ecclesie et translacionis beati antistitis festum annuatim cum maior iubilus celebratur.

$(14)^{96}$ Tanta uero deuocione $i^{97}$ festiuitate ille rex clarissimus cum omni domo sua ob reuerenciam beati Hysidoris conffe $<$ soris $>{ }^{98}$ humilitate ${ }^{99}$ deditus fuisse perhibetur ut, cum peruentum fuisset ad conuiuium, religiosis quibusdam uiris delicatos cibos, deposito regio supercilio, contemptus uice famulorum manibus propriis apponere $<t>100$. Regina quoque cum filiis et filiabus suis reliquaque multitudine $\mathrm{e}^{101}$ more seruulorum omne obsequium humiliter impendebant.

$(15)^{102}$ In eo autem loco, quo sancti corporis reliquie a fideli plebe uenerantur, tanta et talia miracula, que ${ }^{103}$ Dominus noster Ihesus Christus ad honorem et gloriam sui nominis est dignatus ostendere, cecis lumen restituendo, surdis auditum reformando, inmundos spiritus ab obsessis corporibus eiciendo, claudis gressum reintegrando, ut omnes talia Dei magnalia audientes uerterentur in quoddam iubilum et stuporem, sed consuetudo more partim impericia, partim negligencia sunt obtecta.

5. (1) ${ }^{104}$ Ysidori doctoris egregii et Hyspalensis archiepiscopi mirabilia ex multis paucissima huic operi compingentes, primo est inter mirabilia conputandum quod cum ciuitas Yspalensis propter ${ }^{105}$ calidum equoris uaporem numquam sit solita uredinem perpeti glaciei ${ }^{106}$, eo tamen anno quo exinde membra beata sunt abstracta, adeo urente glacie eius est exusta ${ }^{107}$ quod neque in uineis neque in oliuetis seu ficetis aliquis fructus remanserit. In quo quidem significari uidetur elementa discessum sancti corporis persensisse.

$(2)^{108}$ Item cum nuncii regis peruenirent ad portam ciuitatis Legionis, orta est in populo sedicio in quo loco beatissimi Hysidori reuerendi Aluiti episcopi deberent corpora reppelliri. Super qua contencione in abbatem Siliacensem unanimiter concordantes, precibus fusis ad Dominum et ad doctorem Ysidorum, utriusque corpora iussit gestatorio subponi. Et in introitu ciuitatis uirga equos feriens et angelico ducatu[i $]^{109}$ comitentes, corpus Aluiti uenerabilis equs ad ecclesiam almiflue Matris Christi, equi uero, qui corpus beati Hysidori defferebant, a uocatu angelico ipsum ad ecclesiam beati Iohannis Babtiste diuinitus detulerunt, $<\ldots . .>^{110}$ ad

\footnotetext{
${ }^{96}$ Source : Leg. 7.

${ }^{97}$ in scripsi cum fonte, et $L$.

${ }^{98}$ conffesoris scripsi cum fonte, conffe $L$.

${ }^{99}$ humilitate : humilitati Leg. 7.

${ }^{100}$ apponeret scripsi cum fonte, apponere $L$.

${ }^{101}$ reliquaque multitudine : relique multitudini Leg. 7.

${ }^{102}$ Source : Leg. 8.

${ }^{103}$ que non legitur in fonte.

${ }^{104}$ Source : Leg. 9.

${ }^{105}$ propter scripsi cum fonte, presbyteri $L$.

${ }^{106}$ glaciei scripsi cum fonte, glacialem $L$.

${ }^{107}$ exusta scripsi cum fonte, uita $L$.

${ }^{108}$ Source : hist. trans. 3,3.

${ }^{109}$ ducatu scripsi cum fonte, ducatui $L$.

${ }^{110}$ post detulerunt desunt nonnulla uerba (cf. hist. trans. 3,3 1. 17-26).
} 
ecclesiam almiflue Matris Christi quousque uenerabilis Aluiti corpus fuit traditum sepulture.

(3) ${ }^{111}$ Item Iudeus quidam timore regis Aldeffonsi confugit ad ecclesiam antistitis beatissimi et doctoris. Cumque ibi iaceret uir quidam habens pedes ad nates conuinctos, qui usum membrorum inutilis habebat, a uiris quatuor portabatur. Quem Iudeus intuens, sic alloquitur circunstantes: $\mathrm{Si}$ eger meritis beati Ysidori fuerit liberatus, baptismatis undam suscipiam et huic ecclesie ${ }^{112}$ munera multa dabo. Fusaque oracione ab hiis qui adherant, media nocte, cum omnes se sopori dedissent, eger clammare cepit ita quod omnes surgerent egrumque ${ }^{113}$ assisterent circumquaque.

$(4)^{114}$ Cumque circunstantes eum interrogarent, respondit: Nonne dominum meum Hysidorum uidetis crura mea trahentem, mee egritudini sucurrentem ? Mirati omnes et atoniti audierunt sonitum neruorum, sicuti ligna franguntur. Transactisque aliquantis horis, surgit a lecto, pergit sanus et incolumis ad altare, illudque amplectens lacrimari cepit et ex tunc in Dei ${ }^{15}$ seruicio finaliter supermansit. Que omnia intue $<\mathrm{n}>\mathrm{s}^{116}$, omnia que promiserat adimpleuit. Nam cum omni domo sua est ilico baptizatus pecuniamque ecclesie prefate tradidit et Deo ex tunc seruire studuit et placere.

(5) ${ }^{117}$ Item aliud miraculum. Vir quidam Astoricensis quendam puerum educauerat ${ }^{118}$, qui a natiuitate mutus fuerat atque surdus. Cumque uir predictus Legionem ire decerneret, preffatum puerum secum tulit. Dumque uir ille in uillam ad sua peragenda negocia perrexisset, puer domum exiens casualiter deuenit ad ecclesiam beatissimi conffessoris. Cumque uersus altare pergeret, cuidam uiro induto pontificalibus obuiauit, qui linguam pueri extrahens, ait illi : Vinculis lingue solutus, sanus efficere et gloriam Deo redde.

(6) ${ }^{119}$ Item aliud miraculum. Miles quidam, quem rex feriri decreuerat sentencia capitali, in predicti conffessoris ecclesia se recepit. Quem rex armata manu custodiri iussit et, ne cibus potusue sibi daretur, sub mortis $<$ in $>$ terminacione ${ }^{120}$ prohibuit. Quid plura? VII. reuolutis diebus ad mortis articulum usque miles peruenit. Diuina autem gracia ei minime defuit, ita ut lapis, qui sacerdotis celebrantis subiacet plantis, aquas diuinitus perspicuas emanaret. Ex quibus predictus miles potando sepissime se sufficienter refecit. Que uero superfuerunt, ad honorem Dei et sanctissimi conffessoris et egrorum sanitati usque in presens in uase uitreo conseruantur, adeo dulces et preclare acsi in presenti limpidissimo fonte hauste fuissent.

\footnotetext{
${ }^{111}$ Source : hist. trans. 5,11. 1-13.

${ }^{112}$ ecclesie scripsi cum fonte, ecclesia $L$.

113 egrumque scripsi cum fonte, grabataque $L$.

${ }^{114}$ Source : hist. trans. 5,1 1. 13-24.

115 in dei scripsi, iudei $L$, in eius (sc. Isidori) hist. trans. 5, 1 l. 20.

${ }^{116}$ intuens scripsi, intues $L$.

${ }^{117}$ Source : hist. trans. 5,2 1. 1-10.

${ }^{118}$ educauerat scripsi, aducauerat $L$, nutrierat hist. trans. 5,2 l. 2.

${ }^{119}$ Source : hist. trans. 5,3.

${ }^{120}$ interminacione scripsi cum fonte, terminacione $L$.
} 
(7) ${ }^{121}$ Item cum rege Fernando monacho Hyspanie Arabes Celtiberie ac Carpentanie tributa negarent, ipse rex cum exercitu eos agrediens et uastacionibus infestauit et pristine restituit seruituti. Sed iam uite sibi termino inminente, apparuit ei beatus Ysidorus et diem sui obitus intimauit. Et cum cepisset post modicum infirmari, fecit se Legionem adduci et IX. kalendas ianuarii die sabbati ingressus est ciuitatem, atque ex more corpora sanctorum flexis genibus adorauit petens et supplicans ut, cum dies obitus iam instaret, anima eius ab angelis duceretur.

$(8)^{122}$ In ipsa autem nocte dominice Natiuitatis, licet eger, ipse rex cum clericis interffuit matutinis concinens ut infirmitas tollerabat. Atque obseruabatur tunc temporis officium Toletanum. Facto autem die, uocatis episcopis, fecit sibi missam sollempniter celebrari et precepit corporis et sanguinis Domini sacramentum. In crastino autem, uocatis episcopis et abbatibus et uiris religiosis, una cum eis fecit ad ecclesiam se defferri. Et imponito capiti diademate et indutus regalibus indumentis, sarcofago sancti Ysidori clammauit ad Dominum uoce clara : Tua est potencia, tuum regnum, Domine. Tu es super omnes gentes. Tuo imperio omnia sunt subiecta. Quod te donante accipi, restituo tibi regnum. Tantum animam meam in eterna luce iubeas collocari.

$(9)^{123}$ Et his dictis, exutus regalibus ornamentis pro uenia exorabat. Et recepta ab episcopis penitencia et gracia ultime uncionis, indutus cilicio et conperssus cinere duobus diebus in penitencia et lacrimis superuixit. Tertia autem feria, hora $\mathrm{VI}^{\mathrm{a}}$., in festo sancti Iohannis euangeliste, plenus dierum reddidit Deo spiritum inmaculatum et iuxta patrem suum in eadem ecclesia sancti Ysidori est sepultus.

(10) Hucusque fuit ex cronicis Roderici archiepiscopi Toletani et Luce Tudensis episcopi.

(11) ${ }^{124}$ Item in eisdem cronicis continetur quod in ecclesia beati Hysidori apud Legionem in natiuitate sancti Iohannis, hora $\mathrm{VI}^{\mathrm{a}}$., de lapidibus qui erant in altaris gradibus $\mathrm{c}[\mathrm{r}]$ ementati ${ }^{125}$, non ${ }^{126}$ ex terra $<$ sed ex $>^{127}$ substancia $^{128}$ lapidum, cepit aqua cunctis uidentibus emanare et tribus diebus continue emanauit. Erant autem illis diebus in ciuitate Petrus Legionensis episcopus et Pelagius Ouetensis ${ }^{129}$. Qui, audito $^{130}$, cum uniuerso clero et populo ciuitatis induti sacris uestibus ab ecclesia cathedrali usque ad altare sancti Hysidori, ubi hoc acciderat, processerunt. Et peracta missa et sermone proposito ab episcopo Ouetensi $i^{131}$, ad locum miraculi accesserunt et ex ipsa aqua pontifices et ceteri laudantes Deum cum lacrimis potauerunt.

\footnotetext{
${ }^{121}$ Source : hist. reb. 6,13 1. 22-30.

${ }^{122}$ Source : hist. reb. 6,13 1. 31-43.

${ }^{123}$ Source : hist. reb. 6,13 1. 43-54.

${ }^{124}$ Source : hist. reb. 6,34 1. 7-18.

${ }^{125}$ cementati scripsi cum fonte, crementati $L$.

${ }^{126}$ non scripsi cum fonte, uero $L$.

${ }^{127}$ sed ex addidi ex fonte.

${ }^{128}$ substancia scripsi cum fonte, sub aceruo $L$ (ut uidetur).

${ }^{129}$ ouetensis scripsi cum fonte, euetensis $L$.

${ }^{130}$ post audito legitur miraculo in fonte.

${ }^{131}$ ouetensi scripsi cum fonte, euetensi $L$.
} 
$(12)^{132}$ Peracto namque sermone a Pelagio episcopo Ouetensi ${ }^{133}$ et celebrata missa a Petro Legionensi, episcopi et populus uniuersus ex aqua predicta biberunt. Posthec uero statim ${ }^{134}$ fluere cessauerunt. Iusserunt tamen episcopi ut de aqua illa in uasis uitreis seruaretur in memoriam huius facti. Multique infirmi ex ea bibentes curati sunt.

$(13)^{135}$ Videtur autem aqua illa fluxisse miraculose in signum tribulacionum futura $<$ rum $>136$. Nam post hoc prodigium decessit rex nobilis Aldeffonsus apud Toletum, rex quoque Aragonie Aldeffonsus Gallecos in Via de Angos ${ }^{137}$ deuicit ecclesiamque beati Hysidori spoliauit. Quod [cum] ${ }^{138}$ dum fieret, uenit nuncius, quod Sarraceni regnum Aragonie deuastabant. Quibus cum obuiasset, commisso cum eis prelio in loco qui dicitur Fraga, occisus est. Tamen nec uiuus nec mortuus potuit amplius inueniri.

$(14)^{139}$ Item in eisdem cronicis legitur quod, cum rex Aldeffonsus, filius comitis Raymundi et Vrrace regine, filie regis Aldeffonsi, obsedisset Beaciam ${ }^{140}$ ciuitatem et Sarraceni eidem in magna multitudine fortiter restitissent, apparuit eidem regi secum paucos habenti beatus Hysidorus cum dormiret. Et confortans eum dixit se illi datum fuisse ac suo generi a Domino deffenssorem. Et ne in aliquo formidaret Sarracenorum multitudinem hortabatur, sed uiriliter primo mane cum Sarracenis confligeret, quia sicut fumus euanescerent a facie eius et ipsa die eandem caperent ciuitatem.

$(15)^{141}$ Imperator autem tanta uisione confortatus in Domino, primo ${ }^{142}$ inito certamine cum barbaris, statim terga dederunt Sarraceni, nostris relicta innumerabili multitudine spoliorum, statimque Sarraceni eiusdem ciuitatis tradiderunt illi urbem pro uita tantummodo suplicantes. Imperator autem destruxit ipsam et multa castella Maurorum et captiuauit et occidit, ut sibi uisum fuit.

(16) ${ }^{143}$ Posthec reuersus Legionem cum magna gloria, statuit confraternitatem fieri in ipsa ciuitate ob memoriam tanti miraculi et ob honorem sancti Hysidori conffessoris. Reparauit etiam thesauros et hereditates pro parte ${ }^{144}$ magna, quas rex Aragonum et comes Enrricus abstulerant ab ecclesia predicti conffessoris et eandem ecclesiam, que adhuc in iure consistebat regali, consecrauit Domino tradens eam perpetuo iure canonicis regularibus.

\footnotetext{
${ }^{132}$ Source : chron. 4,72 1. 23-30.

${ }^{133}$ ouetensi scripsi cum fonte, euetensi $L$.

${ }^{134}$ post statim legitur aque in fonte.

${ }^{135}$ Sources : uidetur - Toletum chron. 4,72 1. 30-33 et 36-37; rex-deuicit chron. 4,73 1. 12 -

21 ; ecclesiamque - spoliauit chron. 4,72 1.33-35 (cf. ibid. 4,73 1.27-32); quod-inueniri :

chron. 4,73 1. 34-38.

${ }^{136}$ futurarum scripsi, futura $L$.

${ }^{137}$ de angos scripsi cum fonte, clangos $L$.

${ }^{138}$ cum seclusi (cf. chron. 4,73 1. 34).

${ }^{139}$ Source : rex - Aldeffonsi chron. 4,73 1. 1-2; obsedisset - ciuitatem chron. 4,75 1. 9-19.

${ }^{140}$ beaciam : baeciam Chronicon mundi 4,75 l. 9 (sed lectio beatiam legitur in apparatu).

${ }^{141}$ Source : chron. 4,75 1. 19-25.

${ }^{142}$ post primo legitur mane in chron. 4,75 l. 20.

${ }^{143}$ Source : chron. 4,75 1. 26-32.

${ }^{144}$ parte scripsi cum fonte, patre $L$.
} 
$(17)^{145}$ Tandem $^{146}$ illi Dominus graciam et gloriam contulit ut omnes Hyspaniarum reges et principes suo imperio subderentur. Etenim rex Garsias de Nauarra et Reimundus comes Barchinonensis, qui tunc Aragonense regebat regnum, et Sarracenorum ${ }^{147}$, scilicet, Abephandil et Zaphadola, et rex Lupus, uno eodemque tempore eius uasalli fuerunt. In tanto imperii culmine sublimatus, nunquam aliquem subditum ui oppressit, sed multos de thesauris suis gloriose ditauit, et libertates et inmunitates ecclesiis et opressis pauperibus contulit.

$(18)^{148}$ Item in eisdem cronicis legitur quod, cum rex Fernandus esset Benauenti et Petrus Fernandi Castellanus disponeret euertere Ciuitatem Roderici cum Sarracenorum exercitu copioso, apparuit beatus Hysidorus thesaurario monasterii sui, nomine Hysidoro, dicens ut nunciaret regi Fernando aduentum Sarracenorum et uictoriam affuturam. Quod ut factum est, rex Ffernandus occurrens Sarracenis cum eisdem inenarrabilem stratem fecit asistente sibi beato Iacobo et beato Hysidoro.

(19) Qui, tanquam Hyspanie deffenssores, ipsis Christianis in preliis frequenter apparuerunt et ab hostibus deffensauerunt ad laudem almiflui Ihesu Christi, cui est honor et gloria in secula secularum. Amen.

Explicit uita sancti Hysidori.

Jose Carlos Martín

Université de Salamanque

\footnotetext{
${ }^{145}$ Source : chron. 4,75 1. 32-40.

${ }^{146}$ tandem : tantam chron. 4,75 l. 32.

${ }^{147}$ ante sarracenorum legitur reges in fonte.

${ }^{148}$ Source : chron. 4,80 1. 1-14.
} 